\title{
Charge radii and electromagnetic moments of $\mathrm{Li}$ and Be isotopes from the $a b$ initio no-core shell model
}

\author{
C. Forssér $*$ \\ Fundamental Physics, Chalmers University of Technology, 41296 Göteborg, Sweden \\ E. Caurier \\ Institut de Recherches Subatomiques (IN2P3-CNRS-Université Louis Pasteur) \\ Batiment 27/1, 67037 Strasbourg Cedex 2, France \\ P. Navrátil \\ Lawrence Livermore National Laboratory, P.O. Box 808, L-414, Livermore, CA 94551, USA
}

(Dated: October 25, 2018)

\begin{abstract}
Recently, charge radii and ground-state electromagnetic moments of $\mathrm{Li}$ and Be isotopes were measured precisely. We have performed large-scale $a b$ initio no-core shell model calculations for these isotopes using high-precision nucleon-nucleon potentials. The isotopic trends of our computed charge radii, quadrupole and magnetic-dipole moments are in good agreement with experimental results with the exception of the ${ }^{11} \mathrm{Li}$ charge radius. The magnetic moments are in particular well described whereas the absolute magnitudes of the quadrupole moments are about $10 \%$ too small. The small magnitude of the ${ }^{6} \mathrm{Li}$ quadrupole moment is reproduced, and with the CD-Bonn $N N$ potential also its correct sign.

PACS numbers: 21.60.De, 21.10.Ft, 21.10.Ky, 21.30.Fe, 27.20.+n
\end{abstract}

Recent developments of both experimental and theoretical techniques have allowed for very precise measurements of charge radii and ground-state electromagnetic moments of exotic isotopes [1, 2, 3, 4]. In particular, charge radii can be determined through measurements of isotope shifts in certain atomic transitions using highprecision laser spectroscopy at isotope separation facilities [1, 2]. Theoretical calculations of electron correlations, as well as relativistic and QED corrections, can be performed [5, 6] to yield very small uncertainties in the extracted results. Electric quadrupole and magnetic dipole moments can be determined using an experimental method that is based on the nuclear magnetic resonance technique [3, 4].

These observables reflect, in different ways, the evolving nuclear structure along the isotopic chains. Fascinating trends with varying $N / Z$ ratio have been revealed [7]. It is a true challenge for theoretical methods to compute these observables, and to reproduce all of the observed trends simultaneously. In particular, for $a b$ initio manybody methods this type of study allows to test properties of the high-precision nuclear Hamiltonians that are used as the single input to the calculations. But these calculations also constitute a critical test of the limitations of the many-body method itself and of the operators that are used to compute the matrix elements.

Efforts to compute some of these observables with microscopic approaches have been performed using different realistic and semi-realistic interactions $[\underline{8},[9,10,11]$. Concerning the $a b$ initio no-core shell model (NCSM) used in

*christian.forssen@chalmers.se this study, increased computational capabilities and improved algorithms have led to the opportunity to reach significantly extended model spaces. In addition, studies of realistic nuclear Hamiltonians have led some authors to explore the extent to which effects of multi-nucleon forces can be absorbed by non-local terms in the $N N$ interaction [12, 13]. The use of these $N N$ interactions allows, to some extent, to study three-body interaction effects while still maximizing the size of the model space.

In concert, these developments can be regarded as a strong motivation to repeat and extend some earlier NCSM studies [9, 14, 15]. In this Rapid Communication we calculate the charge radii and electromagnetic moments of the $A \leq 11$ chains of $\mathrm{Li}$ and Be isotopes. We compare the performance of two very different $N N$ interactions: (i) the CD-Bonn 2000 interaction (CDB2k) [16], that is a charge-dependent $N N$ interaction based on oneboson exchange; and (2) the INOY IS-M [12] that is a phenomenological interaction for which non-locality was introduced in certain partial waves so that the binding energies of ${ }^{3} \mathrm{H}$ and ${ }^{3} \mathrm{He}$ are described correctly [17]. All calculations are performed up to very large model spaces and efforts are made to quantify the rates of convergence of the observables.

The NCSM method has been described in great detail in several papers, see e.g., Refs. [18, 19, 20]. Here, we just outline the approach as it is applied in the present study. We start from the intrinsic two-body Hamiltonian for the $A$-nucleon system $H_{A}=\mathcal{T}_{\text {rel }}+\mathcal{V}$, where $\mathcal{T}_{\text {rel }}$ is the relative kinetic energy and $\mathcal{V}$ is the sum of two-body nuclear and Coulomb interactions. We solve the many-body problem in a large but finite harmonic-oscillator (HO) basis truncated by a chosen maximal total HO energy of the $A$-nucleon system. If we use realistic nuclear inter- 
actions such as CDB2k or INOY, that generate strong short-range correlations, it is necessary to compute a model-space dependent effective Hamiltonian. For this purpose, we perform a unitary transformation 21, 22] of the Hamiltonian, which accommodates the short-range correlations. In general, the transformed Hamiltonian is an $A$-body operator. Our simplest, yet nontrivial, approximation is to develop a two-particle cluster effective Hamiltonian, while the next improvement is to include three-particle clusters, and so on. The effective interaction is then obtained from the decoupling condition between the model space and the excluded space for the two-nucleon transformed Hamiltonian. Details of the procedure are described in Refs. [18, 19, 23].
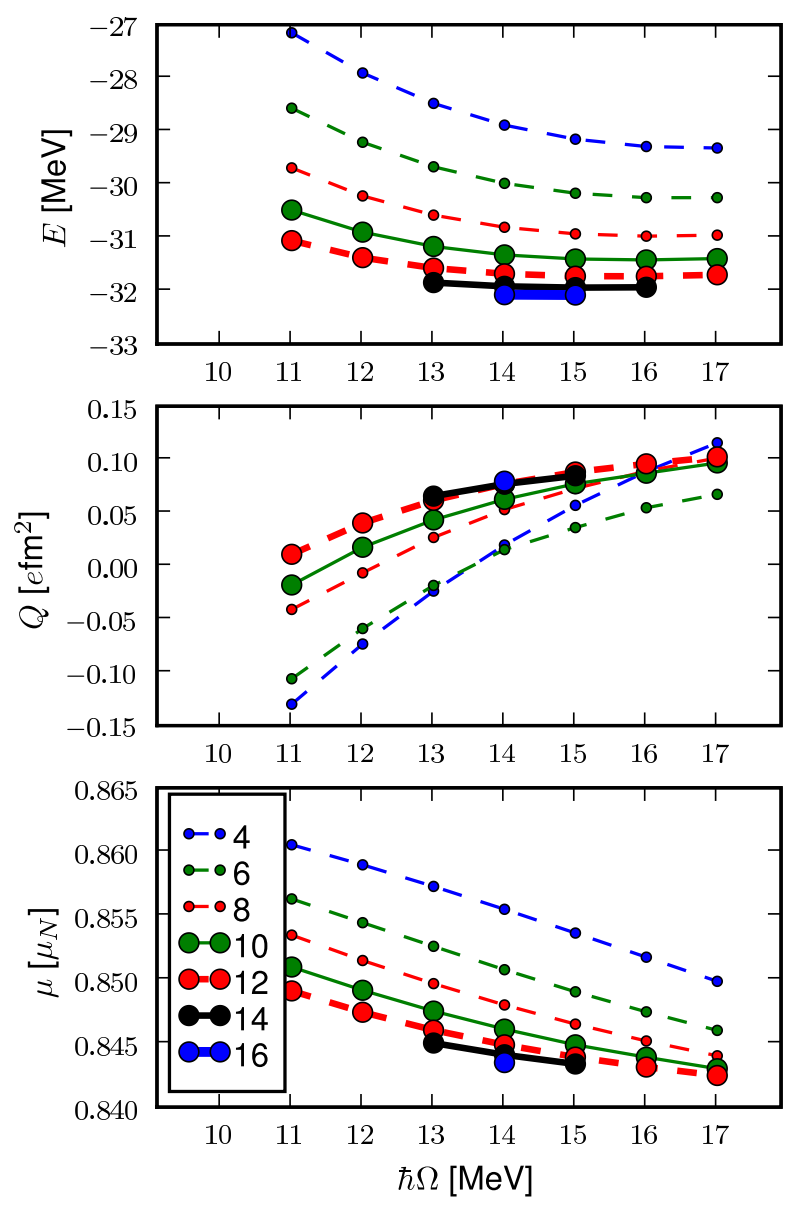

FIG. 1: (Color online) $\hbar \Omega$-dependence for ${ }^{6} \mathrm{Li}$ ground-state observables calculated with the INOY interaction. Each curve corresponds to a particular model space represented by the parameter $N_{\max }$ (see text for details).

In the present application we aim to describe nuclear observables that, in some cases, are sensitive to longrange properties of the wave function. It is therefore essential to include as many terms as possible in the expansion of the total wave function. For this reason, we limit ourselves to the use of realistic two-nucleon $(N N)$ interactions and we only present results obtained with the two-body cluster approximation.

Our calculations are not variational, so higher order terms may contribute with either sign to total binding. Hence, evaluating the dependence on the basis-space parameters help calibrate our convergence. Once the effective interaction is derived, we diagonalize the effective Hamiltonian in a Slater determinant HO basis that spans a complete $N_{\max } \hbar \Omega$ space. This is a highly nontrivial problem because of the very large dimensions we encounter. To solve this problem, we have used a specialized version of the shell model code Antoine 24], adapted to the NCSM [25]. The first step in our study is a search for the optimal HO frequencies. The converged results should not depend on the HO frequency. In practice however, due to the cluster approximation of the effective interaction, our results will be sensitive to the choice of $\hbar \Omega$. Furthermore, by construction the effective interactions depend on the size of the model space, $N_{\max }$, and on the number of nucleons, $A$. In order to investigate these dependences we have performed calculations for a large series of frequencies. As an example, the results from this study for the ${ }^{6} \mathrm{Li}$ ground-state binding energy and electromagnetic moments are presented in Fig. 1 1 for the INOY interaction.

We are looking for the region in which the dependence on $\hbar \Omega$ is the smallest; and we select this frequency (from the calculation in the largest model space) as a starting point for our detailed investigation of ground-state observables. The selected optimal HO frequencies, $\hbar \Omega_{A}$, are presented in Table \.

TABLE I: Selected optimal HO frequencies, $\hbar \Omega_{A}$, (in $\left.[\mathrm{MeV}]\right)$. Also shown is the maximum model space (represented by $N_{\text {max }}$ ) that was reached for each mass number $A$.

\begin{tabular}{c|cccccc}
\hline \hline & \multicolumn{7}{|c}{ Mass number } \\
Interaction & 6 & 7 & 8 & 9 & 10 & 11 \\
\hline CDB2k & 11 & 11 & 12 & 12 & 13 & $13^{a}$ \\
INOY & 14 & 16 & 16 & 16 & 17 & 17 \\
\hline$N_{\max }$ & 16 & 12 & 12 & 10 & 10 & 10 \\
\hline \hline
\end{tabular}

${ }^{a}$ The choice $\hbar \Omega_{A}=12 \mathrm{MeV}$ is used for ${ }^{11} \mathrm{Li}$.

In addition, our results will depend on the size of the model space represented by the parameter $N_{\max }$. Our approach is to fix the HO frequency, at the value $\hbar \Omega_{A}$ specified in Table [ and observe the convergence with increasing model space. At the same time, we investigate the $N_{\text {max }}$-dependence at $\hbar \Omega_{A} \pm 1 \mathrm{MeV}$.

Using these studies we can gauge the degree of convergence of our final results. For a given observable $O$ the total error $\Delta O$ is estimated by

$$
\Delta O=\sqrt{\Delta O_{N}^{2}+\Delta O_{\Omega}^{2}}
$$

where $\Delta O_{N}$ and $\Delta O_{\Omega}$ represent the observed rates of convergence with respect to model-space size and $\mathrm{HO}$ 
frequency, respectively. We choose $\Delta O_{N}$ simply as the difference between the numerical results in the largest and next-to-largest model spaces using the optimal HO frequency. To determine $\Delta O_{\Omega}$ we consider the triplet of results computed at $\hbar \Omega_{A}$ and $\hbar \Omega_{A} \pm 1 \mathrm{MeV}$ in the largest model space for which all three are available. We then define the quantity $\Delta O_{\Omega}$ as the difference between the maximum and minimum value of this triplet of results. The total error $\Delta O$ is presented in the tables of this paper. Ground-state binding energies $(E)$ for $\mathrm{Li}$ isotopes are summarized in Table II. We note that for the

TABLE II: Ground-state binding energies $(E)$ for $\mathrm{Li}$ isotopes. The INOY results are extrapolated energies (with convergence-rate estimates), while the actual computed results from the largest model space are given in square brackets. The experimental results are from [26, 27, 28].

\begin{tabular}{r|cccl}
\hline \hline & \multicolumn{4}{|c}{$E[\mathrm{MeV}]$} \\
& CDB2k & INOY & Exp \\
\hline${ }^{6} \mathrm{Li}$ & $29.07(41)$ & $32.33(19)$ & {$[32.07]$} & 31.99 \\
${ }^{7} \mathrm{Li}$ & $35.56(23)$ & $39.62(40)$ & {$[38.89]$} & 39.24 \\
${ }^{8} \mathrm{Li}$ & $35.82(22)$ & $41.27(51)$ & {$[39.94]$} & 41.28 \\
${ }^{9} \mathrm{Li}$ & $37.88(82)$ & $45.86(74)$ & {$[42.30]$} & 45.34 \\
${ }^{11} \mathrm{Li}$ & $37.72(45)$ & $42.50(95)^{a}$ & {$[40.44]$} & $45.72(1)$ \\
\hline \hline
\end{tabular}

${ }^{a}$ The exponential convergence rate is not fully reached.

INOY NN potential, the ground-state energy convergence is very uniform with respect to the HO frequency with systematic changes with $N_{\max }$. The convergence with increasing $N_{\text {max }}$ is quite evident in particular for $A=6-9$, see e.g. the top panel of Fig. 1] and we are able to extrapolate assuming an exponential dependence on $N_{\max }$ as $E\left(N_{\max }\right)=E_{\infty}+a \exp \left(-b N_{\max }\right)$. The same type of extrapolation was successfully performed for the $\mathrm{He}$ isotopes [29]. Therefore, extrapolated INOY energies are given in Table I together with computed results from the largest model space (in square brackets).

It also deserves to be mentioned that we can, in principle, have different $\hbar \Omega$-dependence for different observables. In particular since we're using effective Hamiltonians to compute the eigenenergies and eigenfunctions but are employing bare operators to evaluate other observables. In practice, however, the choice of $\hbar \Omega_{A}$ is relatively stable. The exception to this general rule is observed for those observables that depend critically on the size of the system. In particular, for large systems we sometimes observe a different $\hbar \Omega$-dependence for the electric quadrupole moment and for the radius. In this case the convergence-rate estimate should be viewed as providing the magnitude of variations around the maximum model space reached. The effective-operator formalism for general one- and two-body operators was developed and studied in Ref. [30], finding weak renormalization of long-range operators.

To complete the present survey we have collected results for odd- $A$ Be isotopes from earlier papers. The
${ }^{7}$ Be results are partly from Ref. [14] while ${ }^{9,11}$ Be results are from Ref. [15]. However, additional calculations were performed in the present study to provide convergence rate estimates. In addition, new calculations have been performed for the ${ }^{10} \mathrm{Be}$ ground state.

Ground-state quadrupole and magnetic dipole moments have been calculated in the impulse approximation using bare operators. Two-body meson-exchange currents have been shown to increase isovector magnetic moments [31, 32] but are not included here. The $N_{\max }{ }^{-}$ dependence of $Q$ and $\mu$ is presented in Fig. 2 for odd $\mathrm{Li}$ isotopes using the CDB2k interaction. We note that the magnetic moment is usually very well converged in the NCSM. The quadrupole moment is sensitive to the length scale of the NCSM basis and for smaller model spaces it tends to increase with decreasing HO frequency. Final results for all isotopes are presented in Table III to-

TABLE III: Ground-state quadrupole moments $(Q)$, magnetic dipole moments $(\mu)$, and charge radii $\left(r_{c}\right)$ for $\mathrm{Li}$ and Be isotopes. Theoretical results for ${ }^{7} \mathrm{Be}$ and ${ }^{9,11} \mathrm{Be}$ are partly from Refs. 14, 15]. The experimental results are from Refs. 3, 4, 26, 27] for electromagnetic moments, and from Refs. [1, 2, 6] for radii.

\begin{tabular}{|c|c|c|c|}
\hline & \multicolumn{3}{|c|}{$Q\left[\mathrm{e} \mathrm{fm}^{2}\right]$} \\
\hline & CDB2k & INOY & Exp \\
\hline${ }^{6} \mathrm{Li}$ & $-0.066(40)$ & $+0.080(19)$ & $-0.0806(6)$ \\
\hline${ }^{7} \mathrm{Li}$ & $-3.20(22)$ & $-2.79(17)$ & $-4.00(3)$ \\
\hline${ }^{8} \mathrm{Li}$ & $+2.78(12)$ & $+2.55(12)$ & $+3.14(2)$ \\
\hline${ }^{9} \mathrm{Li}$ & $-2.66(22)$ & $-2.30(12)$ & $-3.06(2)$ \\
\hline${ }^{11} \mathrm{Li}$ & $-2.81(27)$ & $-2.32(13)$ & $-3.33(5)$ \\
\hline${ }^{7} \mathrm{Be}$ & $-5.50(48)$ & $-4.68(28)$ & - \\
\hline \multirow[t]{3}{*}{${ }^{9} \mathrm{Be}$} & $+4.12(26)$ & $+3.67(23)$ & $+5.288(38)$ \\
\hline & \multicolumn{3}{|c|}{$\mu\left[\mu_{N}\right]$} \\
\hline & CDB2k & INOY & $\operatorname{Exp}$ \\
\hline${ }^{6} \mathrm{Li}$ & $+0.843(5)$ & $+0.843(2)$ & +0.822 \\
\hline${ }^{7} \mathrm{Li}$ & $+3.01(2)$ & $+3.02(2)$ & +3.256 \\
\hline${ }^{8} \mathrm{Li}$ & $+1.24(6)$ & $+1.42(4)$ & +1.654 \\
\hline${ }^{9} \mathrm{Li}$ & $+2.89(2)$ & $+2.98(5)$ & +3.437 \\
\hline${ }^{11} \mathrm{Li}$ & $+3.56(4)$ & $+3.54(4)$ & $+3.671(1)$ \\
\hline${ }^{7} \mathrm{Be}$ & $-1.14(1)$ & $-1.15(1)$ & $-1.3995(5)$ \\
\hline${ }^{9} \mathrm{Be}$ & $-1.22(9)$ & $-1.06(6)$ & -1.1774 \\
\hline \multirow[t]{3}{*}{${ }^{11} \mathrm{Be}$} & $-1.55(6)$ & $-1.47(3)$ & $-1.6813(5)$ \\
\hline & \multicolumn{3}{|c|}{$r_{c}[\mathrm{fm}]$} \\
\hline & CDB2k & INOY & Exp \\
\hline${ }^{6} \mathrm{Li}$ & $2.40(6)$ & $2.29(4)$ & $2.540(28)$ \\
\hline${ }^{7} \mathrm{Li}$ & $2.36(7)$ & $2.20(5)$ & $2.390(30)$ \\
\hline${ }^{8} \mathrm{Li}$ & $2.31(8)$ & $2.16(5)$ & $2.281(32)$ \\
\hline${ }^{9} \mathrm{Li}$ & $2.25(10)$ & $2.07(6)$ & $2.185(33)$ \\
\hline${ }^{11} \mathrm{Li}$ & $2.26(13)$ & $2.06(6)$ & $2.426(34)$ \\
\hline${ }^{7} \mathrm{Be}$ & $2.56(10)$ & $2.39(5)$ & $2.647(17)$ \\
\hline${ }^{9} \mathrm{Be}$ & $2.41(11)$ & $2.22(6)$ & $2.519(12)$ \\
\hline${ }^{10} \mathrm{Be}$ & $2.34(9)$ & $2.16(6)$ & $2.357(18)$ \\
\hline${ }^{11} \mathrm{Be}$ & $2.37(11)$ & $2.19(7)$ & $2.463(16)$ \\
\hline
\end{tabular}

gether with recent and very precise experimental results using the nuclear magnetic resonance technique [3, 4 ] . We note that the two different interactions used in this study give very similar isotopic trends but with a consistently smaller magnitude for the INOY interaction. This observation is connected to the anomalously large nuclear density generated by this interaction found already in 
${ }^{4} \mathrm{He}$ calculations 29, 33]. For ${ }^{6} \mathrm{Li}$ the quadrupole moment is basically converged and the magnitude agrees well with the experimental value. However, for the larger systems the calculated magnitudes are consistently $\gtrsim 10 \%$ too small as compared to the experimental results. This result is expected from working in a finite $\mathrm{HO}$ basis and we observe that the magnitude of the quadrupole moment is steadily increasing with increasing model space, as shown in Fig. 2.

The point-nucleon radii $\left(r_{p t-p}\right.$ and $\left.r_{p t-n}\right)$ can be computed from the NCSM wave functions with complete removal of spurious CM motion. We have translated these results into a rms charge radius $\left(r_{c}\right)$ by folding in proton $\left\langle R_{p}^{2}\right\rangle$ and neutron $\left\langle R_{n}^{2}\right\rangle$ rms charge radii [34] and adding the Darwin-Foldy term [35] according to the relation $\left\langle r_{c}^{2}\right\rangle=\left\langle r_{p t-p}^{2}\right\rangle+\left\langle R_{p}^{2}\right\rangle+N\left\langle R_{n}^{2}\right\rangle / Z+3 \hbar^{2} /\left(4 M_{p}^{2} c^{2}\right)$. These results are compared to recent high-precision laser
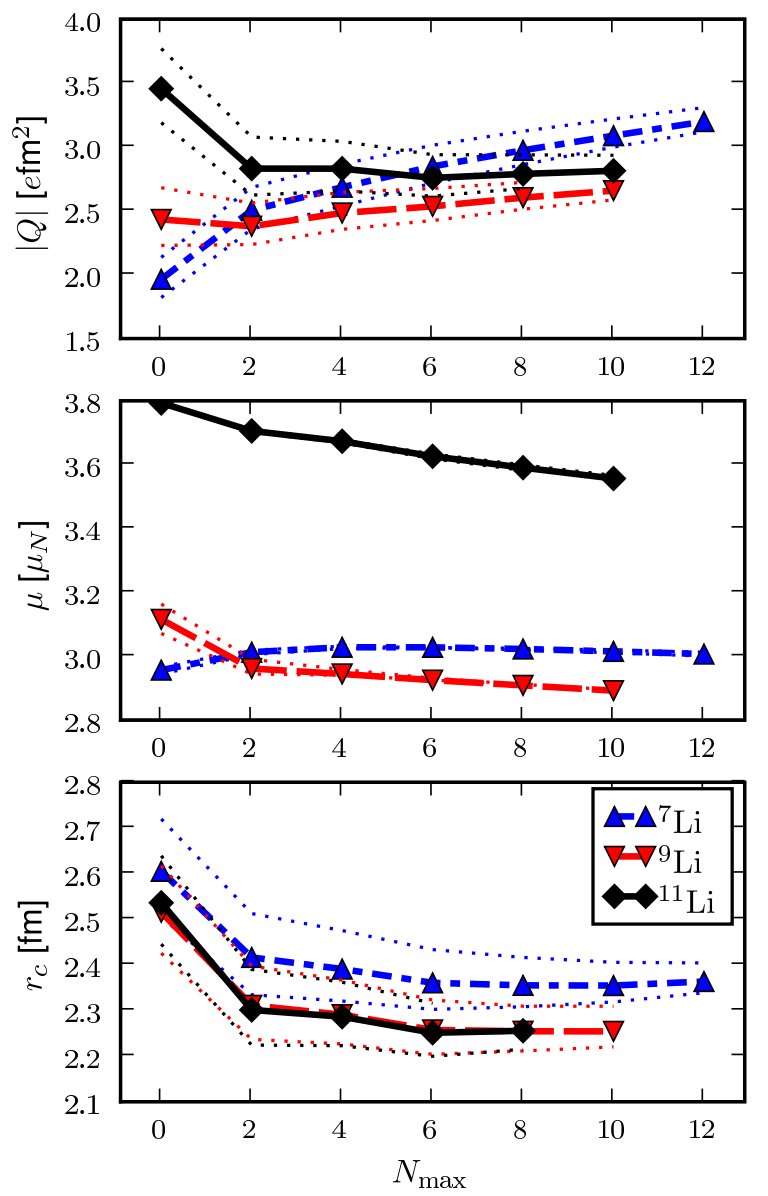

FIG. 2: (Color online) $N_{\max }$-dependence of ground-state electromagnetic moments and charge radius for odd Li isotopes calculated with the CDB2k interaction. Each curve corresponds to a fixed HO frequency. In particular, thick (thin) lines correspond to $\hbar \Omega_{A}\left(\hbar \Omega_{A} \pm 1 \mathrm{MeV}\right)$ for each isotope.

spectroscopy measurements [1, 2] in Table III] The experimental results are obtained using previously mea- sured ${ }^{7} \mathrm{Li}$ and ${ }^{9} \mathrm{Be}$ radii as references. The convergence of the calculated radius with increasing $N_{\max }$ is shown in Fig. 2 for odd $\mathrm{Li}$ isotopes using the CDB2k interaction. We also conclude that the larger binding energy obtained with the INOY interaction can partially be explained by an abnormally large nuclear density and consequently a charge radius that is too small. For CDB2k, the radius results are rather well converged and show good agreement with experiment. However, the known halo structure of the ${ }^{11} \mathrm{Li}$ ground-state is not reproduced in the limited HO model space of the NCSM.

In summary we have computed charge radii and electomagnetic moments of $\mathrm{Li}$ and $\mathrm{Be}$ isotopes using two different, high-precision nuclear Hamiltonians within the $a b$ initio NCSM. In Fig. 3 we compare the calculated
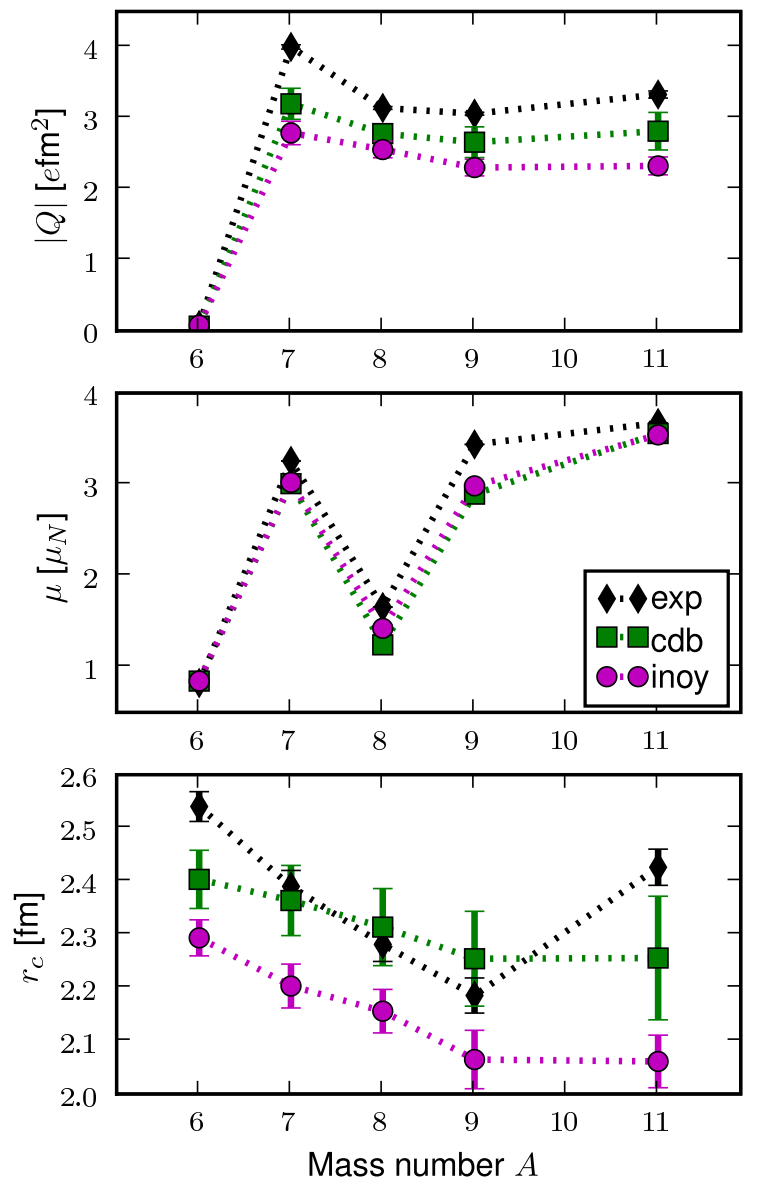

FIG. 3: (Color online) NCSM calculated electric quadrupole moments, magnetic dipole moments, and charge radii of $\mathrm{Li}$ isotopes compared with experimental results. See also Table III

and experimental trends for these observables for the $\mathrm{Li}$ chain of isotopes. With the exception of the radius of the ${ }^{11} \mathrm{Li}$ halo ground-state we find a very good agreement between NCSM results and recent experiments. The overall trends of all observables are well reproduced. Magnetic 
dipole moments are usually characterized by very good convergence properties in the NCSM and we find a good agreement with the experimental values. Another success is the tiny quadrupole moment of ${ }^{6} \mathrm{Li}$ that is known to pose a difficult task for most theoretical calculations. In particular, the general failure of three-body models for this observable has been blamed on missing antisymmetrization of the valence nucleons and the nucleons in the alpha-core [36]. The NCSM correctly reproduces the very small value, but with CDB2k and INOY giving different signs. Simultaneously, the trend for the much larger moments of $A=7-11$ is nicely reproduced. We note that the ratio $Q\left({ }^{11} \mathrm{Li}\right) / Q\left({ }^{9} \mathrm{Li}\right)$ is found to be very close to unity, as confirmed recently by very precise experimental data [4]. This finding is obtained without a very accurate description of the dilute halo structure of ${ }^{11} \mathrm{Li}$; a structural feature that we find would require an extension of the HO basis used in the standard NCSM. Still, the decrease of the charge radius of $A=6-9$ iso- topes is reproduced, although the INOY interaction gives too high nuclear densities.

We conclude by observing that the recent achievement of performing very precise measurements of ground-state properties of exotic isotopes proves to be a very valuable tool in the quest for understanding the nuclear interaction, the forms of relevant operators, as well as the evolving structure of the nuclear many-body system.

This research was supported by the Swedish Research Council and the Knut and Alice Wallenberg Foundation. Prepared by LLNL under Contract DE-AC5207NA27344. This work was supported by the LDRD contract No. PLS-09-ERD-020, by the U.S. DOE/SC/NP (Work Proposal Number SCW0498) and by the UNEDF SciDAC Collaboration under DOE grant DE-FC0207ER41457. One of us (C.F.) acknowledges financial support from Stiftelsen Lars Hiertas Minne and from Stiftelsen Längmanska Kulturfonden.
[1] R. Sánchez, W. Nörtershäuser, G. Ewald, D. Albers, J. Behr, P. Bricault, B. A. Bushaw, A. Dax, J. Dilling, M. Dombsky, et al., Phys. Rev. Lett. 96, 033002 (2006).

[2] W. Nörtershäuser, D. Tiedemann, M. Záková, Z. Andjelkovic, K. Blaum, M. L. Bissell, R. Cazan, G. Drake, C. Geppert, M. Kowalska, et al., arXiv:0809.2607v4 [nucl-ex] (2008), Phys. Rev. Lett. (in print).

[3] D. Borremans, D. L. Balabanski, K. Blaum, W. Geithner, S. Gheysen, P. Himpe, M. Kowalska, J. Lassen, P. Lievens, S. Mallion, et al., Phys. Rev. C 72, 044309 (2005).

[4] R. Neugart, D. L. Balabanski, K. Blaum, D. Borremans, P. Himpe, M. Kowalska, P. Lievens, S. Mallion, G. Neyens, N. Vermeulen, et al., Phys. Rev. Lett. 101, 132502 (2008).

[5] Z.-C. Yan and G. W. F. Drake, Phys. Rev. Lett. 91, 113004 (2003).

[6] M. Puchalski and K. Pachucki, Phys. Rev. A 78, 052511 (2008).

[7] B. Jonson, Phys. Rep. 389, 1 (2004).

[8] S. C. Pieper, K. Varga, and R. B. Wiringa, Phys. Rev. C 66, 044310 (2002).

[9] P. Navrátil and B. R. Barrett, Phys. Rev. C 57, 3119 (1998).

[10] K. Varga, Y. Suzuki, and R. G. Lovas, Phys. Rev. C 66, 041302(R) (2002).

[11] Y. Kanada-En'yo and H. Horiuchi, Prog. Theor. Phys. Suppl. 142, 205 (2001).

[12] P. Doleschall, Phys. Rev. C 69, 054001 (2004).

[13] P. Doleschall, I. Borbély, Z. Papp, and W. Plessas, Phys. Rev. C 67, 064005 (2003).

[14] P. Navrátil, C. A. Bertulani, and E. Caurier, Phys. Rev. C 73, 065801 (2006).

[15] C. Forssén, P. Navrátil, W. E. Ormand, and E. Caurier, Phys. Rev. C 71, 044312 (2005).

[16] R. Machleidt, Phys. Rev. C 63, 024001 (2001).

[17] It is important to note that, for this particular version, the on-shell properties of the triplet $P$-wave interactions were modified in order to improve the description of $3 \mathrm{~N}$ analyzing powers.

[18] P. Navrátil, J. P. Vary, and B. R. Barrett, Phys. Rev. Lett. 84(25), 5728 (2000).

[19] P. Navrátil, J. P. Vary, and B. R. Barrett, Phys. Rev. C 62, 054311 (2000).

[20] P. Navrátil, J. P. Vary, W. E. Ormand, and B. R. Barrett, Phys. Rev. Lett. 87, 172502 (2001).

[21] K. Suzuki and S. Y. Lee, Prog. Theor. Phys. 64, 2091 (1980).

[22] K. Suzuki and R. Okamoto, Prog. Theor. Phys. 92, 1045 (1994).

[23] P. Navrátil, G. P. Kamuntavicius, and B. R. Barrett, Phys. Rev. C 61, 044001 (2000).

[24] E. Caurier and F. Nowacki, Acta Phys. Pol. B 30(3), 705 (1999).

[25] E. Caurier, P. Navrátil, W. E. Ormand, and J. P. Vary, Phys. Rev. C 64, 051301(R) (2001).

[26] D. R. Tilley, C. M. Cheves, J. L. Godwin, G. M. Hale, H. M. Hofmann, J. H. Kelley, C. G. Sheu, and H. R. Weller, Nucl. Phys. A 708, 3 (2002).

[27] D. R. Tilley, J. H. Kelley, J. L. Godwin, D. J. Millener, J. E. Purcell, C. G. Sheu, and H. R. Weller, Nucl. Phys. A 745, 155 (2004).

[28] C. Bachelet, G. Audi, C. Gaulard, C. Guénaut, F. Herfurth, D. Lunney, M. de Saint Simon, and C. Thibault, Phys. Rev. Lett. 100, 182501 (2008).

[29] E. Caurier and P. Navrátil, Phys. Rev. C 73, 021302(R) (2006).

[30] I. Stetcu, B. R. Barrett, P. Navrátil, and J. P. Vary, Phys. Rev. C 71, 044325 (2005).

[31] L. E. Marcucci, M. Pervin, S. C. Pieper, R. Schiavilla, and R. B. Wiringa, Phys. Rev. C 78, 065501 (2008).

[32] R. Schiavilla, V. G. J. Stoks, W. Glöckle, H. Kamada, A. Nogga, J. Carlson, R. Machleidt, V. R. Pandharipande, R. B. Wiringa, A. Kievsky, et al., Phys. Rev. C 58, 1263 (1998).

[33] R. Lazauskas and J. Carbonell, Phys. Rev. C 70, 044002 (2004).

[34] C. Amsler et al., Phys. Lett. B 667, 1 (2008), (Particle 
Data Group).

[35] J. L. Friar, J. Martorell, and D. W. L. Sprung, Phys. Rev. A 56, 4579 (1997).
[36] M. Unkelbach and H. M. Hofmann, Few-Body Syst. 11, 143 (1991). 